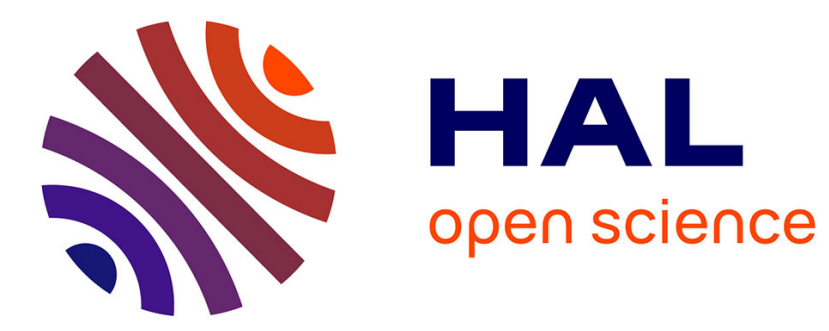

\title{
On a divisor problem related to the Epstein Zeta-Function, III
}

Guangshi Lü, Jie Wu, Wenguang Zhai

\section{To cite this version:}

Guangshi Lü, Jie Wu, Wenguang Zhai. On a divisor problem related to the Epstein Zeta-Function, III. Quarterly Journal of Mathematics, 2012, 63 (4), pp.953-963. hal-01278402

\section{HAL Id: hal-01278402 \\ https://hal.science/hal-01278402}

Submitted on 24 Feb 2016

HAL is a multi-disciplinary open access archive for the deposit and dissemination of scientific research documents, whether they are published or not. The documents may come from teaching and research institutions in France or abroad, or from public or private research centers.
L'archive ouverte pluridisciplinaire HAL, est destinée au dépôt et à la diffusion de documents scientifiques de niveau recherche, publiés ou non, émanant des établissements d'enseignement et de recherche français ou étrangers, des laboratoires publics ou privés. 


\title{
ON A DIVISOR PROBLEM RELATED TO THE EPSTEIN ZETA-FUNCTION, III
}

\author{
GUANGSHI LÜ, JIE WU \& WENGUANG ZHAI
}

\begin{abstract}
In this paper we study the mean square of the error term $\Delta_{k}^{*}(Q, x)$ in a divisor problem related to the Epstein zeta-function. An asymptotic formula has been obtained when $k=2$.
\end{abstract}

\section{INTRODUCTION}

This is the third part of our series of papers on a divisor problem related to the Epstein zeta-function [10,11]. First we recall some notation there. Let $\ell \geqslant 2$, $\mathbf{y}:=\left(y_{1}, \ldots, y_{\ell}\right)$ and $\mathbf{A}=\left(a_{i j}\right)$ be an integral matrix such that $a_{i i} \equiv 0(\bmod 2)$ for $0 \leqslant i \leqslant \ell$. Thus a positive definite quadratic form $Q(\mathbf{y})$ can be written as

$$
Q(\mathbf{y})=\frac{1}{2} \mathbf{y}^{\mathrm{t}} \mathbf{A} \mathbf{y}=\sum_{1 \leqslant i<j \leqslant \ell} a_{i j} y_{i} y_{j}+\frac{1}{2} \sum_{1 \leqslant i \leqslant \ell} a_{i i} y_{i}^{2},
$$

where $\mathbf{y}^{\mathbf{t}}$ is the transpose of $\mathbf{y}$. The corresponding Epstein zeta-function is initially defined by the Dirichlet series

$$
Z_{Q}(s):=\sum_{\mathbf{y} \in \mathbb{Z}^{\ell} \backslash\{\mathbf{0}\}} Q(\mathbf{y})^{-s}=\sum_{n \geqslant 1} a_{n} n^{-s} \quad(\Re e s>\ell / 2),
$$

where $a_{n}$ is the number of the solutions of the equation $Q(\mathbf{y})=n$ with $\mathbf{y} \in \mathbb{Z}^{\ell}$. It is known that $Z_{Q}(s)$ has an analytic continuation to the whole complex plane $\mathbb{C}$ with only a simple pole at $s=\ell / 2$, and satisfies a functional equation of Riemann type (cf. [13]). For each integer $k \geqslant 1$, we define $a_{k}(n)$ by

$$
Z_{Q}(s)^{k}=\sum_{n \geqslant 1} a_{k}(n) n^{-s} \quad(\Re e s>\ell / 2)
$$

and put

$$
\Delta_{k}^{*}(Q, x):=\sum_{n \leqslant x} a_{k}(n)-x^{\ell / 2} P_{k}(\log x),
$$

where $P_{k}(\log x):=x^{-\ell / 2} \operatorname{Res}_{s=\ell / 2}\left(Z_{Q}(s)^{k} x^{s} s^{-1}\right)$ is a polynomial of $\log x$ of degree $k-1$. The study on asymptotic behavior of the error term $\Delta_{k}^{*}(Q, x)$ has received

Date: December 5, 2015.

2000 Mathematics Subject Classification. 11F30, 11F11,11F66.

Key words and phrases. Epsetin zeta-function, divisor problem, modular form.

Guangshi Lü is supported in part by key project of the National Natural Science Foundation of China (Grant No. 11031004), Shandong Province Natural Science Foundation (Grant No. ZR2009AM007), and NCET. Wenguang Zhai is supported by the Natural Science Foundation of Beijing (Grant No. 1112010). 
much attention $[8,1,13]$. In particular Sankaranarayanan [13] showed that for $k \geqslant 2$ and $\ell \geqslant 3$,

$$
\Delta_{k}^{*}(Q, x) \ll x^{\ell / 2-1 / k+\varepsilon},
$$

where and throughout this paper $\varepsilon$ denotes an arbitrarily small positive constant. Recently inspired by Iwaniec's book [6], Lü [10] marked that (1.4) can been improved for the quadratic forms of level one (see [6, Chapter 11]). These quadratic forms are defined by $Q(\mathbf{y})=\frac{1}{2} \mathbf{y}^{\mathrm{t}} \mathbf{A} \mathbf{y}$ verifying the following supplementary conditions:

$$
\ell \equiv 0(\bmod 8), \quad \mathbf{A} \text { is equivalent to } \mathbf{A}^{-1}, \quad \operatorname{det}(\mathbf{A})=1 .
$$

Denote by $\mathcal{Q}_{\ell}$ the set of such quadratic forms. For $Q \in \mathcal{Q}_{\ell}$, we have [6, (11.32)]

$$
a_{n}=A_{\ell} \sigma_{\ell / 2-1}(n)+a_{f}(n, Q) \quad(n \geqslant 1)
$$

where

$$
A_{\ell}:=\frac{(2 \pi)^{\ell / 2}}{\zeta(\ell / 2) \Gamma(\ell / 2)}, \quad \sigma_{k}(n)=\sum_{d \mid n} d^{k},
$$

$\zeta(s)$ is the Riemann zeta-function, $\Gamma(s)$ is the Gamma function and $a_{f}(n, Q)$ is the $n$th Fourier coefficient of a cusp form $f(z, Q)$ of weight $\ell / 2$ with respect to the full modular group $\mathrm{SL}(2, \mathbb{Z})$. Thus

$$
Z_{Q}(s)=A_{\ell} \zeta(s) \zeta(s-\ell / 2+1)+L(s, f) \quad(\Re e s>\ell / 2),
$$

where $L(s, f)$ is the Hecke $L$-function associated with $f(z, Q)$. According to Deligne's well known work [2], we know

$$
\left|a_{f}(n, Q)\right| \leqslant n^{(\ell / 2-1) / 2} \tau(n) \quad(n \geqslant 1),
$$

where $\tau(n)$ is the divisor function. With the help of these properties, Lü [10] (for $k \geqslant 4$ ) and Lü, Wu \& Zhai [11] (for $k=2,3$ ) obtained

$$
\Delta_{k}^{*}(Q, x) \ll x^{\ell / 2-1+\theta_{k}+\varepsilon},
$$

where $\theta_{k}$ is the exponent in the classical $k$-dimension divisor problem

$$
\Delta_{k}(x):=\sum_{n \leqslant x} \tau_{k}(n)-\operatorname{Res}_{s=1}\left(\zeta(s)^{k} x^{s} s^{-1}\right) \ll x^{\theta_{k}+\varepsilon} \quad(x \geqslant 2) .
$$

In particular we can take $\theta_{2}=131 / 416$ [4], $\theta_{3}=43 / 96$ [7] and $\theta_{k}=(k-1) /(k+2)$ for $k \geqslant 4[15]$. Besides, an $\Omega$-result has been established in [11]: if $8 \mid \ell$ and $Q(\mathbf{y}) \in \mathcal{Q}_{\ell}$, then we have for $k=2,3$ that

$$
\Delta_{k}^{*}(Q, x)=\Omega\left(x^{\ell / 2-1+(k-1) / 2 k}(\log x)^{(k-1) /(2 k)}\left(\log _{2} x\right)^{a}\left(\log _{3} x\right)^{-b^{\prime}}\right),
$$

where $a=\frac{k+1}{2 k}\left(k^{(2 k) /(k+1)}-1\right), b^{\prime}$ is any constant greater than $\frac{3 k-1}{4 k}$ and $\log _{r}$ denotes the $r$-fold iterated logarithm.

The aim of this paper is to study the mean square of $\Delta_{k}^{*}(Q, x)$.

Theorem 1. If $8 \mid \ell$, then for any quadratic form $Q(\mathbf{y}) \in \mathcal{Q}_{\ell}$, we have

$$
\int_{1}^{T}\left|\Delta_{2}^{*}(Q, x)\right|^{2} \mathrm{~d} x=C_{\ell} T^{\ell-1 / 2}+O\left(T^{\ell-1}(\log T)^{3} \log _{2} T\right)
$$


where

$$
g_{a}(n):=\sum_{d \mid n} \frac{\tau(d) \tau(n / d)}{d^{a}}, \quad C_{\ell}:=\frac{3 A_{\ell}^{4}}{(2 \ell-1) \pi^{2}} \sum_{n=1}^{\infty} \frac{g_{(\ell-3) / 2}(n)^{2}}{n^{3 / 2}}
$$

The estimate $O\left(T^{\ell-1}(\log T)^{3} \log _{2} T\right)$ follows from the result of [9] on the mean square of $\Delta_{2}(x)$.

Theorem 2. For $k \geqslant 2,8 \mid \ell$ and $Q(\mathbf{y}) \in \mathcal{Q}_{\ell}$, we define

$$
\begin{aligned}
& \beta_{k}:=\inf \left\{b_{k}: \int_{1}^{T}\left|\Delta_{k}(x)\right|^{2} \mathrm{~d} x \ll T^{1+2 b_{k}+\varepsilon}\right\}, \\
& \beta_{k}^{*}:=\inf \left\{b_{k}^{*}: \int_{1}^{T}\left|\Delta_{k}^{*}(Q, x)\right|^{2} \mathrm{~d} x \ll T^{\ell-1+2 b_{k}^{*}+\varepsilon}\right\} .
\end{aligned}
$$

Then $\beta_{k}^{*}=\beta_{k}$. Further we have $\beta_{k}^{*} \geqslant(k-1) / 2 k$ and the equality holds if the Lindelöf hypothesis of $\zeta(s)$ is true.

Ivić $[5$,$] proved that$

$$
\beta_{3}=1 / 3, \quad \beta_{4}=3 / 8, \quad \beta_{5} \leqslant 119 / 260, \quad \beta_{6} \leqslant 1 / 2, \quad \beta_{7} \leqslant 39 / 70 .
$$

According to Theorem 2, the same estimates for $\beta_{k}^{*}$ hold.

Acknowledgement. The authors deeply thank the referee for valuable comments and suggestions.

\section{An Expression of $\Delta_{2}^{*}(Q, x)$}

In [11], we actually established the formula

$$
\Delta_{2}^{*}(Q, x)=A_{\ell}^{2} x^{\ell / 2-1} \sum_{d \leqslant x} \frac{\tau(d)}{d^{\ell / 2-1}} \Delta_{2}\left(\frac{x}{d}\right)+O\left(x^{\ell / 2-1+\varepsilon}\right) .
$$

From it we can deduce $\Omega$-result of $\Delta_{2}^{*}(Q, x)$. However, it is not enough to prove Theorem 1. So first we will give a better expression of $\Delta_{2}^{*}(Q, x)$.

Lemma 2.1. If $8 \mid \ell$, then for any quadratic form $Q(\mathbf{y}) \in \mathcal{Q}_{\ell}$, we have

$$
\begin{aligned}
\Delta_{2}^{*}(Q, x)= & A_{\ell}^{2} x^{\ell / 2-1} \sum_{d \leqslant x} \frac{\tau(d)}{d^{\ell / 2-1}}\left(\Delta_{2}\left(\frac{x}{d}\right)-\frac{1}{4}\right) \\
& -2 A_{\ell} x^{\ell / 2-1} \sum_{d \leqslant x} \frac{b(d)}{d^{\ell / 2-1}} \psi\left(\frac{x}{d}\right)+O\left(x^{\ell / 2-5 / 4}\right),
\end{aligned}
$$

where $\psi(t):=\{t\}-\frac{1}{2}$ and $\{t\}$ denotes the fractional part of $t$.

Proof. From (1.5) we have

$$
Z_{Q}(s)^{2}=A_{\ell}^{2} \zeta(s)^{2} \zeta(s-\ell / 2+1)^{2}+A_{\ell} \zeta(s) \zeta(s-\ell / 2+1) L(s, f)+L(s, f)^{2} .
$$


Here the last two terms do not appear when $\ell=8,16$ since there are no cusp forms of weights 4 and 8 with respect to $\mathrm{SL}(2, \mathbb{Z})$. Thus we can write

$$
\begin{aligned}
\sum_{n \leqslant x} a_{2}(n)= & A_{\ell}^{2} \sum_{d \leqslant x} \tau(d) \sum_{m \leqslant x / d} \tau(m) m^{\ell / 2-1} \\
& +2 A_{\ell} \sum_{d \leqslant x} b(d) \sum_{m \leqslant x / d} m^{\ell / 2-1}+\sum_{d \leqslant x} c(d)
\end{aligned}
$$

where $b(n)$ and $c(n)$ are defined by

$$
\zeta(s) L(s, f)=\sum_{n=1}^{\infty} b(n) n^{-s} \quad \text { and } \quad L(s, f)^{2}=\sum_{n=1}^{\infty} c(n) n^{-s}
$$

for $\Re e s>\ell / 2$, respectively. By using Deligne's bound (1.6), it is easy to see that

$$
|b(n)| \leqslant n^{(\ell / 2-1) / 2} \tau_{3}(n) \quad \text { and } \quad|c(n)| \leqslant n^{(\ell / 2-1) / 2} \tau_{4}(n) .
$$

Thus

$$
\sum_{n \leqslant x}(|b(n)|+|c(n)|) \ll x^{\ell / 4+1 / 2}(\log x)^{3}
$$

By partial summation we have

$$
\begin{aligned}
\sum_{m \leqslant x} \tau(m) m^{\ell / 2-1}= & \frac{2}{\ell} x^{\ell / 2}\left(\log x-\frac{2}{\ell}+2 \gamma\right)+x^{\ell / 2-1} \Delta_{2}(x) \\
& -(\ell / 2-1) \int_{1}^{x} \Delta_{2}(t) t^{\ell / 2-2} \mathrm{~d} t
\end{aligned}
$$

By using Voronoï's well known formula [16]:

$$
\int_{1}^{t} \Delta_{2}(u) \mathrm{d} u=\frac{t}{4}+O\left(t^{3 / 4}\right)
$$

a simple partial summation leads to

$$
(\ell / 2-1) \int_{1}^{x} \Delta_{2}(t) t^{\ell / 2-2} \mathrm{~d} t=\frac{1}{4} x^{\ell / 2-1}+O\left(x^{\ell / 2-5 / 4}\right) .
$$

Combining these, we find that

$$
\begin{aligned}
\sum_{m \leqslant x} \tau(m) m^{\ell / 2-1}= & \frac{2}{\ell} x^{\ell / 2}\left(\log x-\frac{2}{\ell}+2 \gamma\right) \\
& +x^{\ell / 2-1}\left(\Delta_{2}(x)-\frac{1}{4}\right)+O\left(x^{\ell / 2-5 / 4}\right)
\end{aligned}
$$

Similarly (even easier)

$$
\sum_{m \leqslant x} m^{\ell / 2-1}=\frac{2}{\ell} x^{\ell / 2}-x^{\ell / 2-1} \psi(x)+O\left(x^{\ell / 2-2}\right) .
$$

Now the required result follows from (2.1), (2.3), (2.4) and (2.5). 


\section{Proof of Theorem 1}

\subsection{Beginning of the Proof. Let}

$$
\widetilde{\Delta}_{2}^{*}(Q, x):=\frac{\Delta_{2}^{*}(Q, x)}{A_{\ell}^{2} x^{\ell / 2-1}} \quad \text { and } \quad \widetilde{C}_{\ell}:=\frac{1}{\pi^{2}} \sum_{n=1}^{\infty} \frac{g_{(\ell-3) / 2}(n)^{2}}{n^{3 / 2}} .
$$

Clearly it is sufficient to prove that

$$
\int_{1}^{T}\left|\widetilde{\Delta}_{2}^{*}(Q, x)\right|^{2} \mathrm{~d} x=\frac{\widetilde{C}_{\ell}}{6} T^{3 / 2}+O\left(T(\log T)^{3} \log _{2} T\right) .
$$

According to Lemma 2.1, we can write

$$
\widetilde{\Delta}_{2}^{*}(Q, x)=U(x)-V(x)+O\left(x^{\ell / 2-5 / 4}\right),
$$

where

$$
U(x):=\sum_{d \leqslant x} \frac{\tau(d)}{d^{\ell / 2-1}}\left(\Delta_{2}\left(\frac{x}{d}\right)-\frac{1}{4}\right), \quad V(x):=\frac{2}{A_{\ell}} \sum_{d \leqslant x} \frac{b(d)}{d^{\ell / 2-1}} \psi\left(\frac{x}{d}\right) .
$$

Next we shall prove

$$
\begin{aligned}
& \int_{1}^{T} U^{2}(x) \mathrm{d} x=\frac{\widetilde{C}_{\ell}}{6} T^{3 / 2}+O\left(T(\log T)^{3} \log _{2} T\right), \\
& \int_{1}^{T} U(x) V(x) \mathrm{d} x \ll T(\log T)^{2},
\end{aligned}
$$

which imply (3.1).

3.2. Preparation. In this subsection, we shall prove some preliminary estimates, which are useful later.

Lemma 3.1. Let $a>0, b>1, \ell>a+b$ and $A \geqslant 1$. We have

$$
\sum_{\substack{d_{1}, d_{2} \leqslant T \\ m_{1}, m_{2} \leqslant M \\ d_{1} m_{2}=d_{2} m_{1}}} \frac{\tau\left(d_{1}\right) \tau\left(d_{2}\right) \tau\left(m_{1}\right) \tau\left(m_{2}\right)}{\left(d_{1} d_{2}\right)^{\ell / 2-a / 2}\left(m_{1} m_{2}\right)^{b / 2}}=\sum_{n=1}^{\infty} \frac{g_{(\ell-a-b) / 2}(n)^{2}}{n^{b}}+O_{A}\left(\frac{(\log T)^{3}}{T^{b-1}}\right),
$$

$$
\sum_{\substack{d_{1}, d_{2} \leqslant T \\ m_{1}, m_{2} \leqslant M \\ d_{1} m_{2} \neq d_{2} m_{1}}} \frac{\tau\left(d_{1}\right) \tau\left(d_{2}\right) \tau\left(m_{1}\right) \tau\left(m_{2}\right)}{\left(d_{1} d_{2}\right)^{\ell / 2-3 / 4}\left(m_{1} m_{2}\right)^{3 / 4}} \frac{1}{\mid \sqrt{m_{1} / d_{1}}-\sqrt{m_{2} / d_{2} \mid}} \ll_{A}(\log T)^{3} \log _{2} T,
$$

$$
\sum_{\substack{d_{1}, d_{2} \leqslant T \\ m_{1}, m_{2} \leqslant M}} \frac{\tau\left(d_{1}\right) \tau\left(d_{2}\right) \tau\left(m_{1}\right) \tau\left(m_{2}\right)}{\left(d_{1} d_{2}\right)^{\ell / 2-3 / 4}\left(m_{1} m_{2}\right)^{3 / 4}} \frac{1}{\sqrt{m_{1} / d_{1}}+\sqrt{m_{2} / d_{2}}} \ll_{A}(\log T)^{3} \log _{2} T .
$$

uniformly for $1 \leqslant T \leqslant M \leqslant T^{A}$, where $g_{r}(n)$ is defined as in (1.7). 
Proof. First we write

$$
\begin{aligned}
S_{1}(T, M) & =\sum_{n \leqslant T M} \frac{1}{n^{b}}\left(\sum_{\substack{d \leqslant T ; m \leqslant M \\
d m=n}} \frac{\tau(d) \tau(m)}{d^{(\ell-a-b) / 2}}\right)^{2} \\
& =\sum_{n=1}^{\infty} \frac{g_{(\ell-a-b) / 2}(n)^{2}}{n^{b}}+O\left(\sum_{n>T} \frac{g_{(\ell-a-b) / 2}(n)^{2}}{n^{b}}\right) .
\end{aligned}
$$

It is easy to see that $g_{r}(n)$ is multiplicative, $g_{r}(p)=2+2 / p^{r}$ and $g_{r}\left(p^{\nu}\right) \ll_{r}(\nu+1)$ for all $p$ and $\nu \geqslant 1$. Applying Theorem 2.1 of [14] with $x=y$ and $\kappa=4$ to $g_{r}(n)^{2}$ leads to the following inequality

$$
\sum_{n \leqslant x} g_{r}(n)^{2} \ll_{r} x(\log x)^{3} \quad(r>0, x \geqslant 2) .
$$

From it and (3.7), we can easily deduce (3.4).

Similarly we can write

$$
S_{2}(T, M) \leqslant \sum_{\substack{n, n^{\prime} \leqslant T M \\ n \neq n^{\prime}}} \frac{g_{\ell / 2-2}(n) g_{\ell / 2-2}\left(n^{\prime}\right)}{\left(n n^{\prime}\right)^{3 / 4}} \frac{1}{\left|\sqrt{n}-\sqrt{n^{\prime}}\right|} \ll_{A}(\log T)^{3} \log _{2} T .
$$

In the last step we have used the bound of Lau \& Tsang [9].

The estimate (3.6) is an immediate consequence of (3.4) with $a=b=2$ and (3.5) if noting that

$$
\frac{1}{\sqrt{m_{1} / d_{1}}+\sqrt{m_{2} / d_{2}}} \ll \begin{cases}\left(\frac{d_{1} d_{2}}{m_{1} m_{2}}\right)^{1 / 4} & \text { if } m_{1} / d_{1}=m_{2} / d_{2} \\ \frac{1}{\left|\sqrt{m_{1} / d_{1}}-\sqrt{m_{2} / d_{2}}\right|} & \text { if } m_{1} / d_{1} \neq m_{2} / d_{2} .\end{cases}
$$

3.3. Proof of (3.2). According to Meurman [12], we have

$$
\Delta_{2}(x)-\frac{1}{4}=\frac{x^{1 / 4}}{\sqrt{2} \pi} \sum_{m \leqslant M} \frac{\tau(m)}{m^{3 / 4}} \cos \left(4 \pi \sqrt{x m}-\frac{\pi}{4}\right)+E(x)
$$

for all $M>x>1$, where

$$
E(x) \ll \begin{cases}x^{-1 / 4} & \text { if }\|x\| \geqslant x^{5 / 2} M^{-1 / 2}, \\ x^{\varepsilon} & \text { if }\|x\| \leqslant x^{5 / 2} M^{-1 / 2} .\end{cases}
$$

Thus we can write, with the choice of $M=T^{10}>x$,

$$
U(x)=A(x)+B(x)
$$

where

$$
\begin{aligned}
& A(x):=\frac{x^{1 / 4}}{\sqrt{2} \pi} \sum_{d \leqslant x} \frac{\tau(d)}{d^{\ell / 2-3 / 4}} \sum_{m \leqslant M} \frac{d(m)}{m^{3 / 4}} \cos \left(4 \pi \sqrt{\frac{m}{d} x}-\frac{\pi}{4}\right), \\
& B(x):=\sum_{d \leqslant x} \frac{\tau(d)}{d^{\ell / 2-1}} E\left(\frac{x}{d}\right) .
\end{aligned}
$$


In view of the identity $2 \cos u \cos v=\cos (u-v)+\cos (u+v)$, we easily see that

$$
A(x)^{2}=A_{1}(x)+A_{2}(x)+A_{3}(x),
$$

where

$$
\begin{aligned}
& A_{1}(x):=\frac{x^{1 / 2}}{4 \pi^{2}} \sum_{\substack{d_{1}, d_{2} \leqslant x \\
m_{1}, m_{2} \leqslant M \\
m_{1} d_{2}=m_{2} d_{1}}} \frac{\tau\left(d_{1}\right) \tau\left(d_{2}\right) \tau\left(m_{1}\right) \tau\left(m_{2}\right)}{\left(d_{1} d_{2}\right)^{\ell / 2-3 / 4}\left(m_{1} m_{2}\right)^{3 / 4}}, \\
& A_{2}(x):=\frac{x^{1 / 2}}{4 \pi^{2}} \sum_{\substack{d_{1}, d_{2} \leqslant x \\
m_{1}, m_{2} \leqslant M \\
m_{1} d_{2} \neq m_{2} d_{1}}} \frac{\tau\left(d_{1}\right) \tau\left(d_{2}\right) \tau\left(m_{1}\right) \tau\left(m_{2}\right)}{\left(d_{1} d_{2}\right)^{\ell / 2-3 / 4}\left(m_{1} m_{2}\right)^{3 / 4}} \cos \left(4 \pi\left(\sqrt{\frac{m_{1}}{d_{1}}}-\sqrt{\frac{m_{2}}{d_{2}}}\right) \sqrt{x}\right), \\
& A_{3}(x):=\frac{x^{1 / 2}}{4 \pi^{2}} \sum_{\substack{d_{1}, d_{2} \leqslant x \\
m_{1}, m_{2} \leqslant M}} \frac{\tau\left(d_{1}\right) \tau\left(d_{2}\right) \tau\left(m_{1}\right) \tau\left(m_{2}\right)}{\left(d_{1} d_{2}\right)^{\ell / 2-3 / 4}\left(m_{1} m_{2}\right)^{3 / 4}} \cos \left(4 \pi\left(\sqrt{\frac{m_{1}}{d_{1}}}+\sqrt{\frac{m_{2}}{d_{2}}}\right) \sqrt{x}\right) .
\end{aligned}
$$

By using (3.4) we have

$$
\begin{aligned}
\int_{1}^{T} A_{1}(x) \mathrm{d} x & =\frac{1}{4 \pi^{2}} \sum_{\substack{d_{1}, d_{2} \leqslant T \\
m_{1}, m_{2} \leqslant M \\
m_{1} d_{2}=m_{2} d_{1}}} \frac{\tau\left(d_{1}\right) \tau\left(d_{2}\right) \tau\left(m_{1}\right) \tau\left(m_{2}\right)}{\left(d_{1} d_{2}\right)^{\ell / 2-3 / 4}\left(m_{1} m_{2}\right)^{3 / 4}} \int_{\max \left\{d_{1}, d_{2}\right\}}^{T} x^{1 / 2} \mathrm{~d} x \\
& =\frac{\widetilde{C}_{\ell}}{6} T^{3 / 2}+O\left(T(\log T)^{3}\right) .
\end{aligned}
$$

With the help of the first derivative test and (3.5), we get

$$
\begin{aligned}
\int_{1}^{T} A_{2}(x) \mathrm{d} x & \leqslant \sum_{1 \leqslant k \leqslant 2 \log T}\left|\int_{T / 2^{k}}^{T / 2^{k-1}} A_{2}(x) \mathrm{d} x\right| \\
& \ll \sum_{1 \leqslant k \leqslant 2 \log T}\left(T / 2^{k}\right) S_{2}\left(T / 2^{k-1}, M\right) \\
& \ll T(\log T)^{3} \log _{2} T .
\end{aligned}
$$

Similarly we have

$$
\int_{1}^{T} A_{3}(x) \mathrm{d} x \ll T .
$$

Combining these estimates, we find that

$$
\int_{1}^{T} A(x)^{2} \mathrm{~d} x=\frac{\widetilde{C}_{\ell}}{6} T^{3 / 2}+O\left(T(\log T)^{3} \log _{2} T\right) .
$$

By Cauchy's inequality, it follows

$$
B(x)^{2} \leqslant \sum_{d \leqslant x} \frac{\tau(d)^{2}}{d^{2}} \sum_{d \leqslant x} \frac{1}{d^{\ell-4}} E\left(\frac{x}{d}\right)^{2} \ll \sum_{d \leqslant x} \frac{1}{d^{\ell-4}} E\left(\frac{x}{d}\right)^{2},
$$


which combining (3.9) allows us to deduce that

$$
\begin{aligned}
\int_{1}^{T} B(x)^{2} \mathrm{~d} x & \ll \sum_{d \leqslant T} \frac{1}{d^{\ell-5}}\left(\int_{\|t\| \leqslant t^{5 / 2} M^{-1 / 2}}^{T / d} t^{\varepsilon} \mathrm{d} t+\int_{\|t\| \geqslant t^{5 / 2} M^{-1 / 2}}^{T / d} t^{-1 / 2} \mathrm{~d} t\right) \\
& \ll \sum_{d \leqslant T} \frac{1}{d^{\ell-5}}\left\{\left(\frac{T}{d}\right)^{7 / 2+\varepsilon} \frac{1}{M^{1 / 2}}+\left(\frac{T}{d}\right)^{1 / 2}\right\} \\
& \ll T^{1 / 2}
\end{aligned}
$$

From (3.11) and (3.12), we get, via Cauchy's inequality, that

$$
\int_{1}^{T} A(x) B(x) \mathrm{d} x \ll T .
$$

Now the asymptotic formula (3.2) follows from (3.10), (3.11), (3.12) and (3.13).

3.4. Proof of (3.3). By using Theorem 4.5 in Graham and Kolesnik [3]

$$
\Delta_{2}(u)=-2 \sum_{m \leqslant \sqrt{u}} \psi(u / m)+O(1)
$$

and (2.2), we have

$$
\int_{1}^{T} U(x) V(x) \mathrm{d} x \ll \sum_{d \leqslant T} \frac{\tau(d)}{d^{\ell / 2-1}} \sum_{m \leqslant(T / d)^{1 / 2}} \sum_{n \leqslant T} \frac{\tau_{3}(n)}{n^{\ell / 4-1 / 2}}|I(d, m, n)|+T,
$$

where

$$
I(d, m, n):=\int_{\max \left\{d m^{2}, n\right\}}^{T} \psi\left(\frac{x}{d m}\right) \psi\left(\frac{x}{n}\right) \mathrm{d} x .
$$

For $\psi(u)$, it is well-known that the finite Fourier expansion

$$
\psi(u)=-\sum_{1 \leqslant h \leqslant H} \frac{\sin (2 \pi h u)}{\pi h}+O\left(\min \left\{1, \frac{1}{H\|u\|}\right\}\right)
$$

holds for any $H \geqslant 2$. It is easily seen that for any $r>0$

$$
\begin{aligned}
\int_{\max \left\{d m^{2}, n\right\}}^{T} \min \left\{1, \frac{1}{H\|x / r\|}\right\} \mathrm{d} x & =r \int_{m^{2}}^{T / r} \min \left\{1, \frac{1}{H\|t\|}\right\} \mathrm{d} t \\
& \ll T \int_{0}^{1 / 2} \min \left\{1, \frac{1}{H t}\right\} \mathrm{d} t \\
& \ll T H^{-1} \log H .
\end{aligned}
$$

From these we deduce

$$
I(d, m, n) \ll \sum_{h_{1}, h_{2} \leqslant H} \frac{\left|I\left(h_{1}, h_{2}\right)\right|}{h_{1} h_{2}}+\frac{T(\log H)^{2}}{H}
$$


where

$$
\begin{aligned}
I\left(h_{1}, h_{2}\right) & :=\int_{\max \left\{d m^{2}, n\right\}}^{T} \sin \left(\frac{2 \pi h_{1} x}{d m}\right) \sin \left(\frac{2 \pi h_{2} x}{n}\right) \mathrm{d} x \\
& \ll \begin{cases}1 /\left|h_{1} / d m-h_{2} / n\right| & \text { if } h_{1} n \neq h_{2} d m, \\
T & \text { if } h_{1} n=h_{2} d m .\end{cases}
\end{aligned}
$$

Here we have used the identity $2 \sin u \sin v=\cos (u-v)-\cos (u+v)$ and the first derivative test when $h_{1} n \neq h_{2} d m$.

Inserting (3.15) into (3.14), we get

$$
\int_{1}^{T} U(x) V(x) \mathrm{d} x \ll T S_{4}(T, H)+S_{5}(T, H)+T+\frac{T^{3 / 2}(\log H)^{2}}{H},
$$

where

$$
\begin{aligned}
S_{4}(T, H) & :=\sum_{d \leqslant T} \frac{\tau(d)}{d^{\ell / 2-1}} \sum_{m \leqslant(T / d)^{1 / 2}} \sum_{n \leqslant T} \frac{\tau_{3}(n)}{n^{\ell / 4-1 / 2}} \sum_{\substack{h_{1}, h_{2} \leqslant H \\
h_{1} n=h_{2} d m}} \frac{1}{h_{1} h_{2}} \\
& \leqslant \sum_{r \leqslant H T} \frac{1}{r^{2}} \sum_{n \mid r} \frac{\tau_{3}(n)}{n^{\ell / 4-3 / 2}} \sum_{h_{2} d m=r} \frac{\tau(d) m}{d^{\ell / 2-2}} \ll \sum_{r \leqslant H T} \frac{1}{r} \ll \log (H T)
\end{aligned}
$$

and

$$
\begin{aligned}
S_{5}(T, H) & :=\sum_{d \leqslant T} \frac{\tau(d)}{d^{\ell / 2-1}} \sum_{m \leqslant(T / d)^{1 / 2}} \sum_{n \leqslant T} \frac{\tau_{3}(n)}{n^{\ell / 4-1 / 2}} \sum_{\substack{h_{1}, h_{2} \leqslant H \\
h_{1} n \neq h_{2} d m}} \frac{d m n}{h_{1} h_{2}\left|h_{1} n-h_{2} d m\right|} \\
& =\sum_{\substack{r_{1}, r_{2} \leqslant H T \\
r_{1} \neq r_{2}}} \frac{1}{r_{1} r_{2}\left|r_{1}-r_{2}\right|} \sum_{\substack{h_{2} \leqslant H, d \leqslant T, m \leqslant(T / d)^{1 / 2} \\
h_{2} d m=r_{1}}} \frac{\tau(d)(d m)^{2}}{d^{\ell / 2-1}} \sum_{\substack{n \leqslant T, h_{1} \leqslant H \\
h_{1} n=r_{2}}} \frac{\tau_{3}(n)}{n^{\ell / 4-5 / 2}} \\
& \leqslant T \sum_{\substack{r_{1}, r_{2} \leqslant H T \\
r_{1} \neq r_{2}}} \frac{1}{r_{1} r_{2}\left|r_{1}-r_{2}\right|} \sum_{h_{2} d m=r_{1}} \frac{\tau(d)}{d^{\ell / 2-2}} \sum_{h_{1} n=r_{2}} \frac{\tau_{3}(n)}{n^{\ell / 4-5 / 2}} \\
& \ll T \sum_{|r| \leqslant H T} \frac{1}{|r|} \sum_{r_{2} \leqslant H T} \frac{1}{r_{2}} \\
& \ll T(\log H T)^{2} .
\end{aligned}
$$

This proves (3.3) with the choice of $H=T$.

\section{Proof of Theorem 2}

For each $r \geqslant 2$, let $\delta_{r}$ and $\delta_{r}^{*}$ denote the infimum of $\sigma>0$ such that

$$
\int_{-\infty}^{\infty} \frac{|\zeta(\sigma+\mathrm{i} t)|^{r}}{|\sigma+\mathrm{i} t|^{2}} \mathrm{~d} t \ll 1 \quad \text { and } \quad \int_{-\infty}^{\infty} \frac{\left|Z_{Q}(\sigma+\mathrm{i} t)\right|^{r}}{|\sigma+\mathrm{i} t|^{2}} \mathrm{~d} t \ll 1,
$$

respectively. According to [5, Lemma 13.1], we have

$$
\beta_{k}=\delta_{2 k}
$$


On the other hand, following the proof of this lemma word by word by replacing $\zeta(s)$ by $Z_{Q}(s)$ and $\Delta_{k}(x)$ by $\Delta_{k}^{*}(Q, x)$ respectively, we can prove

$$
\beta_{k}^{*}+\ell / 2-1=\delta_{2 k}^{*}
$$

Finally it is easy to see that

$$
|\zeta(s-\ell / 2+1)| \ll\left|Z_{Q}(s)\right| \ll|\zeta(s-\ell / 2+1)|
$$

for $\ell / 2-1 \leqslant \sigma \leqslant \ell / 2$. Thus

$$
\delta_{r}^{*}=\ell / 2-1+\delta_{r} .
$$

Now Theorem 2 follows from (4.1), (4.2) and (4.3) by noting that the Lindelöf hypothesis implies $\delta_{r}=1 / 2-1 / r$ for any $r \geqslant 2$.

\section{REFERENCES}

[1] K. Chandrasekharan \& R. Narasimhan, Functional equations with multiple gamma factors and the average order of arithmetical functions, Ann. of Math. 76 (1962), 93-136.

[2] P. Deligne, La Conjecture de Weil, Inst. Hautes Études Sci. Publ. Math. 43 (1974), 29-39.

[3] S. W. Graham \& G. Kolesnik, Van der Corput's Method of Exponential Sums, Cambridge University Press, London Math. Soc. Lecture Note Ser. 126, 1991.

[4] M. N. Huxley, Integer points, exponential sums and the Riemann zeta function, in: Number theory for the millennium, II (Urbana, IL, 2000), 275-290, A K Peters, Natick, MA, 2002.

[5] A. Ivić, The Riemann zeta-function, John Wiley \& Sons, New York, Chichester, Brisbane, Toronto, Singapore, 1985.

[6] H. Iwaniec, Topics in classical automorphic forms, Graduate Studies in Mathematics, vol. 17, American Mathematical Society, Providence, Rhode Island, 1997.

[7] G. Kolesnik, On the estimation of multiple exponential sums, in: Recent progress in analytic number theory, Vol. 1 (Durham, 1979), Academic Press (London-New York, 1981), 231-246.

[8] E. Landau, Über die Anzahl der Gitterpunkte in gewissen Bereichen, Göttinger Nachr. 18 (1912), 687-773.

[9] Y.-K. Lau \& K.-M. Tsang, On the mean square formula of the error term in the Dirichlet divisor problem, Math. Proc. Camb. Phil. Soc., Vol. 146 (2009), 277-287.

[10] Guangshi Lü, On a divisor problem related to the Epstein zeta-function, Bull. London Math. Soc. 42 (2010), 267-274.

[11] Guangshi Lü, Jie Wu \& Wenguang Zhai, On a divisor problem related to the Epstein zetafunction, II, J. Number Theory, 131(2011), no. 9, 1734-1742.

[12] T. Meurman, On the mean square of the Riemann zeta-function, Quart. J. Math. Oxford Ser. (2) 38 (1987), no. 151, 337-343.

[13] A. Sankaranarayanan, On a divisor problem related to the Epstein zeta-function, Arch. Math. 65 (1995), 303-309.

[14] G. Tenenbaum \& J. Wu, Moyennes de certaines fonctions multiplicatives sur les entiers friables, J. Reine Angew. Math. 564 (2003), 119-167.

[15] E. C. Titchmarsh, The theory of the Riemann zeta-function, Second edition. Edited and with a preface by D. R. Heath-Brown. The Clarendon Press, Oxford University Press, New York, 1986. x+412 pp.

[16] G. F. Voronoï, Sur une fonction transcendante et ses applications à la sommation de quelques séies, Ann. École Normale (3) 21 (1904), 207-267, 459-533.

School of Mathematics, Shandong University, Jinan, Shandong 250100, China

E-mail address: gslv@sdu.edu.cn 
Institut Elie Cartan Nancy, CNRS, Université Henri Poincaré (Nancy 1), inRia, Boulevard des Aiguillettes, B.P. 239, 54506 Vandeuvre-lès-Nancy, France

E-mail address: wujie@iecn.u-nancy.fr

Department of Mathematics, China University of Mining and Technology, BeiJING 100083, CHINA

E-mail address: zhaiwg@hotmail.com 